\title{
Maturation of Widely Distributed Brain Function Subserves Cognitive Development
}

\author{
Beatriz Luna,*` Keith R. Thulborn,ł Douglas P. Munoz,§ Elisha P. Merriam," Krista E. Garver,* \\ Nancy J. Minshew, $†$ Matcheri S. Keshavan,† Christopher R. Genovese,\| \\ William F. Eddy,\| and J ohn A. Sweeney*'† \\ *Neurobehavioral Studies Program, †Department of Psychiatry, ¥MR Research Center, Department of Radiology, "Department \\ of Neuroscience, University of Pittsburgh, Pittsburgh, Pennsylvania; §Department of Physiology, Queen's University, \\ Kingston, Ontario, Canada; and ||Department of Statistics, Carnegie Mellon University, Pittsburgh, Pennsylvania
}

Received J uly 21, 2000; published online March 19, 2001

Cognitive and brain maturational changes continue throughout late childhood and adolescence. During this time, increasing cognitive control over behavior enhances the voluntary suppression of reflexive/impulsive response tendencies. Recently, with the advent of functional MRI, it has become possible to characterize changes in brain activity during cognitive development. In order to investigate the cognitive and brain maturation subserving the ability to voluntarily suppress context-inappropriate behavior, we tested 8-30 year olds in an oculomotor response-suppression task. Behavioral results indicated that adult-like ability to inhibit prepotent responses matured gradually through childhood and adolescence. Functional MRI results indicated that brain activation in frontal, parietal, striatal, and thalamic regions increased progressively from childhood to adulthood. Prefrontal cortex was more active in adolescents than in children or adults; adults demonstrated greater activation in the lateral cerebellum than younger subjects. These results suggest that efficient top-down modulation of reflexive acts may not be fully developed until adulthood and provide evidence that maturation of function across widely distributed brain regions lays the groundwork for enhanced voluntary control of behavior during cognitive development. 2001 Academic Press

Key Words: inhibition; eye movements; cerebellum; neuroimaging.

\section{INTRODUCTION}

Elementary cognitive processes are in place by early childhood; however, the capacity for abstract thought, planning, and cognitive flexibility develop throughout adolescence (Levin et al., 1991). Concurrent with cognitive development are important brain maturational events that continue into early adulthood, including synaptic pruning, elaboration of dendritic arborization
(Huttenlocher, 1990; Changeux et al., 1976), and increased myelination (Yakovlev et al., 1967; Giedd et al., 1999; Paus et al., 1999). It has been traditionally believed that cognitive development during late childhood and adolescence is subserved primarily by the relatively late incorporation of the prefrontal cortex, either by its intrinsic late structural maturation (Bourgeois, 1993; Sowell et al., 1999) or by the maturation of other neocortical regions (Rakic, 1995; Chugani, 1998) that influence their functional integration with prefrontal cortex (Thatcher et al., 1987). I nitial fMRI studies of frontal regions indicate a higher magnitude of activation in the prefrontal cortex in children compared to adults during a Go-No-Go task, providing evidence for a late integration of this area (Casey et al., 1997).

While it is generally accepted that the prefrontal cortex plays a crucial role in higher-order cognition, it is also now well-established that higher-order cognitive functions are subserved by widely distributed and integrated brain systems rather than by the prefrontal cortex independently (Goldman-Rakic, 1988). Hence, it is possible that in conjunction with developmental improvements in function in frontal cortex, there may be increased integration with other brain regions as well. We do not yet know, however, about the maturation of the functional integration of widely distributed circuitry.

Essential to the emergence of adult-level cognition is the ability to voluntarily suppress responses to taskirrelevant information that hamper adaptive information-processing (Dempster, 1993; Bjorklund et al., 1990). The antisaccade task is a paradigm that re quires subjects to vol untarily stop a reflexive eye movement to a prepotent visual stimulus (a novel stimulus in the visual field), and instead move their gaze to the mirror location (Hallett, 1978). It has been used successfully to characterize development of the ability to voluntarily suppress prepotent responses throughout 
late childhood and adolescence (Fischer et al., 1997; Munoz et al., 1998). The brain systems subserving performance on the antisaccade task have been well-delineated in monkeys (Burman et al., 1997; Everling et al., 1999; Funahashi et al., 1993; Gottlieb et al., 1999; Schlag-Rey et al., 1997) and adult humans (Guitton et al., 1985; Sweeney et al., 1996; Muri et al., 1998; Doricchi et al., 1997; O'Driscoll et al., 1995). These areas include the frontal eye field (FEF), supplementary eye fields (SEF), dorsolateral prefrontal cortex (DLPFC), posterior parietal cortex, anterior cingulate cortex, basal ganglia, thalamus, and superior colliculus. It is now possible, using the noninvasive imaging procedure of functional magnetic resonance imaging (fMRI), to characterize the reorganization of brain maturation underlying cognitive development through childhood and adolescence in such widely distributed brain circuitry.

\section{MATERIALS AND METHODS}

\section{Subjects}

Eleven 8- to 13-year-old children (mean $=10.9$ years, $\mathrm{SD}=1.5$, females $=8$ ); 15 14- to 17 -year-old adolescents (mean $=15.7$ years, $S D=1.2$, females $=6$ ); and 10 18- to 30-year-old adults (mean $=24.2$ years, SD = 2.9 , females $=6$ ) participated in the study. All subjects, with the exception of one adult, were right-handed. Additional subjects with head motion over the course of an fMRI study of more than one half of a voxel (five children and one teenager) were excluded. Experimental procedures complied with the Code of Ethics of the World Medical Association (1964 Declaration of Helsinki) and the standards of the University of Pittsburgh Internal Review Board. Subjects and/or their legal guardians provided informed consent after the nature and possible consequences of the studies were explained.

\section{TASKS}

Laboratory eye movement testing was completed on a day prior to brain scanning and included an antisaccade task with 36 trials and a prosaccade (eye movements to visual targets) task with 54 trials, each task lasting approximately 5-10 min. The latency, velocity, amplitude, and duration of eye movements were measured. During the antisaccade task, the number of times subjects erroneously looked at the peripheral cue (prosaccade error rate) was also obtained. This testing assisted in verifying normal oculomotor function and confirming subjects' ability to understand and perform tasks. I mmediately prior to entering the scanner, subjects were familiarized with testing procedures again. Eye movement activity was not monitored in the scanner, but was presumed to be similar to behavioral performance measured in the laboratory, as in other studies (Kastner et al., 1988; Petit et al., 1997, 1999; Corbetta et al., 2000). Additionally, extensive developmental data indicate that the behavior observed in laboratory testing was characteristic of this age period (Fischer et al., 1997; Munoz et al., 1998).

In the scanner, subjects performed a prosaccade vs fixation task to characterize basic sensorimotor control and an antisaccade vs prosaccade task to probe voluntary response suppression. Tasks were presented in a block design. In the prosaccade vs fixation task, subjects fixated a central white crosshair for 6 blocks of $30 \mathrm{~s}$ that alternated with 30 -s blocks of making eye movements to targets appearing in the horizontal axis at an unpredictable location $\left(3^{\circ}, 6^{\circ}\right.$, and $9^{\circ}$ of visual angle to the left and right of center). The antisaccade vs prosaccade task required that subjects look toward the mirror location of a peripheral target during blocks of trials with a red fixation stimulus (antisaccade trials) and look toward the target during trials with a green fixation stimulus (prosaccade trials). As depicted in Fig. 1, all stimuli were white on a black background except for the fixation targets. Prosaccades were used as the comparison condition for the antisaccade task in order to highlight the cognitive rather than sensory and motor components of antisaccade task performance. Our behavioral results demonstrated that subjects performed approximately 2 saccades to acquire the desired locations in the antisaccade task (in order to compensate for prosaccade errors) versus 1.5 saccades in the prosaccade task. Subjects usually make more saccades in antisaccade compared to prosaccade trials because of prosaccade errors and greater difficulty in shifting the eyes to desired locations without sensory guidance. Hence, in the fMRI study, peripheral stimuli were presented every $2.0 \mathrm{~s}$ in the antisaccade task and every $1.5 \mathrm{~s}$ during the prosaccade task. I $n$ this manner, we minimized the possibility that more saccades would be performed in the antisaccade than the prosaccades blocks, which would have increased the contribution of sensorimotor control to brain activation. Subjects performed 8 blocks of prosaccade trials alternating every $36 \mathrm{~s}$ with 7 blocks of antisaccade trials.

fMRI studies were performed on a 3.0 Tesla Signa whole-body MR scanner (General Electric Medical Systems, Milwaukee, WI) with echoplanar imaging capability (Advanced NMR Systems, Inc., Wilmington, MA). Gradient-echo echoplanar imaging was performed using a commercial head RF coil. Acquisition parameters were: TE $=25 \mathrm{~ms}$; TR $=5.0 \mathrm{~s}$; single shot; full k-space; $128 \times 64$ acquisition matrix with a field of view $(\mathrm{FOV})=40 \times 20 \mathrm{~cm}$. In order to cover the whole brain and cerebellum, 23 3-mm-thick oblique slices with a 2-mm gap were aligned to the base of the genu and splenium of the corpus callosum, generating isotropic 3- $\mathrm{mm}^{3}$ voxels. Structural images were obtained 


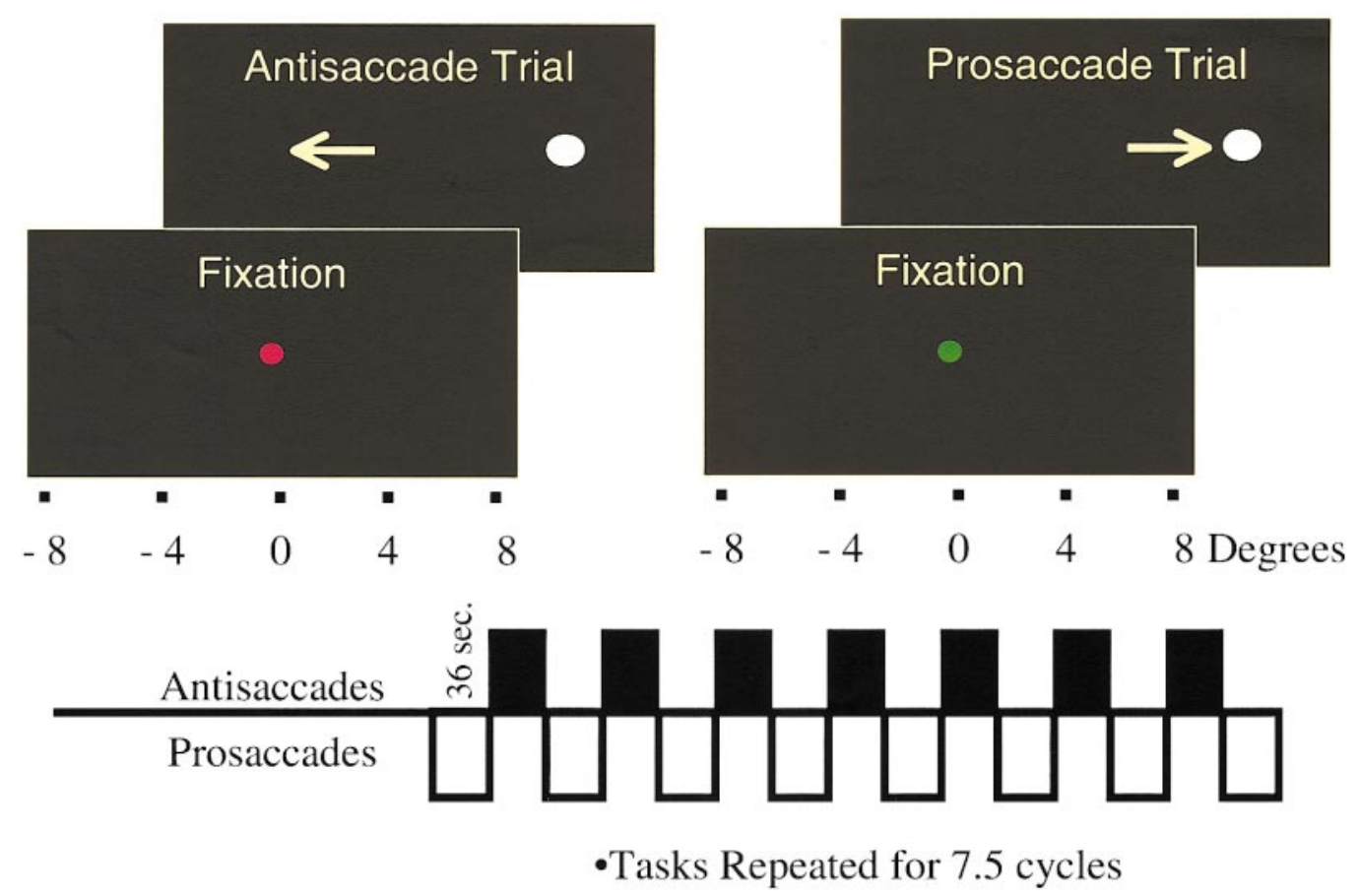

FIG. 1. Stimuli presented during periods of antisaccade trials and prosaccade trials. Each block of trials lasted $36 \mathrm{~s}$. Subjects performed 7.5 cycles of antisaccades alternating with prosaccades every $36 \mathrm{~s}$.

with a 3-D volume spoiled gradient-echo (SPGR) pulse sequence acquired with $1.5-\mathrm{mm}$ slices in the axial plane. Subjects' heads were placed in a RF head coil packed with cushions in order to minimize head motion. Immediately prior to the imaging session, subjects spent approximately 20 min in a simulation scanner that reproduced the sounds and confinement of an MR scanner to acclimate them to the MR environment (Rosenberg et al., 1997).

Fifteen regions of interest ( $\mathrm{ROI}$ ) in each hemisphere were defined: the insula, intraparietal sulcus (IPS), basal ganglia, thalamus, supramarginal gyrus (SMG), dentate nucleus of the cerebellum, lateral cerebellar cortex, superior colliculus (SC), superior precentral sulcus, inferior precentral sulcus, presupplementary motor area (preSMA), SEF, DLPFC, precuneus, and anterior cingulate cortex. Since brain volume is established by school age (Caviness et al., 1996), ROIs were initially drawn on the SPGR of a representative subject that had been normalized to Talairach coordinates using anatomical landmarks. After the template was completed, it was compared to each individual subject's Talairach-transformed SPGR, and the size and shape of ROIs were adjusted to assure that each individual's anatomic ROI was included and that no ROI's overlapped. Analyses were then performed on Talairach transformed brains.

The FEF comprised the full extent of the superior and inferior aspects of the precentral sulcus and were considered separately. The dorsomedial frontal cortex above the cingulate sulcus was divided at the anterior commissure into the immediately caudal SEF and the immediately rostral pre-SMA. DLPFC was defined as the area of the middle frontal gyrus including the inferior and superior frontal sulci, and the gyral surface between them. The anterior cingulate cortex was defined as the cingulate region rostral to the anterior commissure. The precuneus included the area along the interhemispheric fissure, anterior to the parietooccipital sulcus, posterior to the marginal ramus of the cingulate sulcus, and dorsal to the subparietal sulcus. The SMG was defined as the gyrus that wrapped around the posterior tip of the lateral sulcus. The gray matter encompassing the basal ganglia was clearly visible and included the head of the caudate nucleus, putamen, and globus pallidus. The lateral cerebellum was delineated as the cerebellar cortex dorsal to the horizontal fissure. The superior colliculus (SC) included the superior knobs dorsal to the cerebral aqueduct and posterior to the posterior commissure.

\section{ANALYSES}

FIASCO software (Functional Imaging Analysis Software - Computational Olio (Eddy et al., 1996)) was used to apply cubic spline functions to remove slow signal drift, shift the data by $6 \mathrm{~s}$ to compensate for BOLD response recovery functions (Cohen, 1997), and generate functional activation maps based on t tests and percentage BOLD signal change. Multivariate 


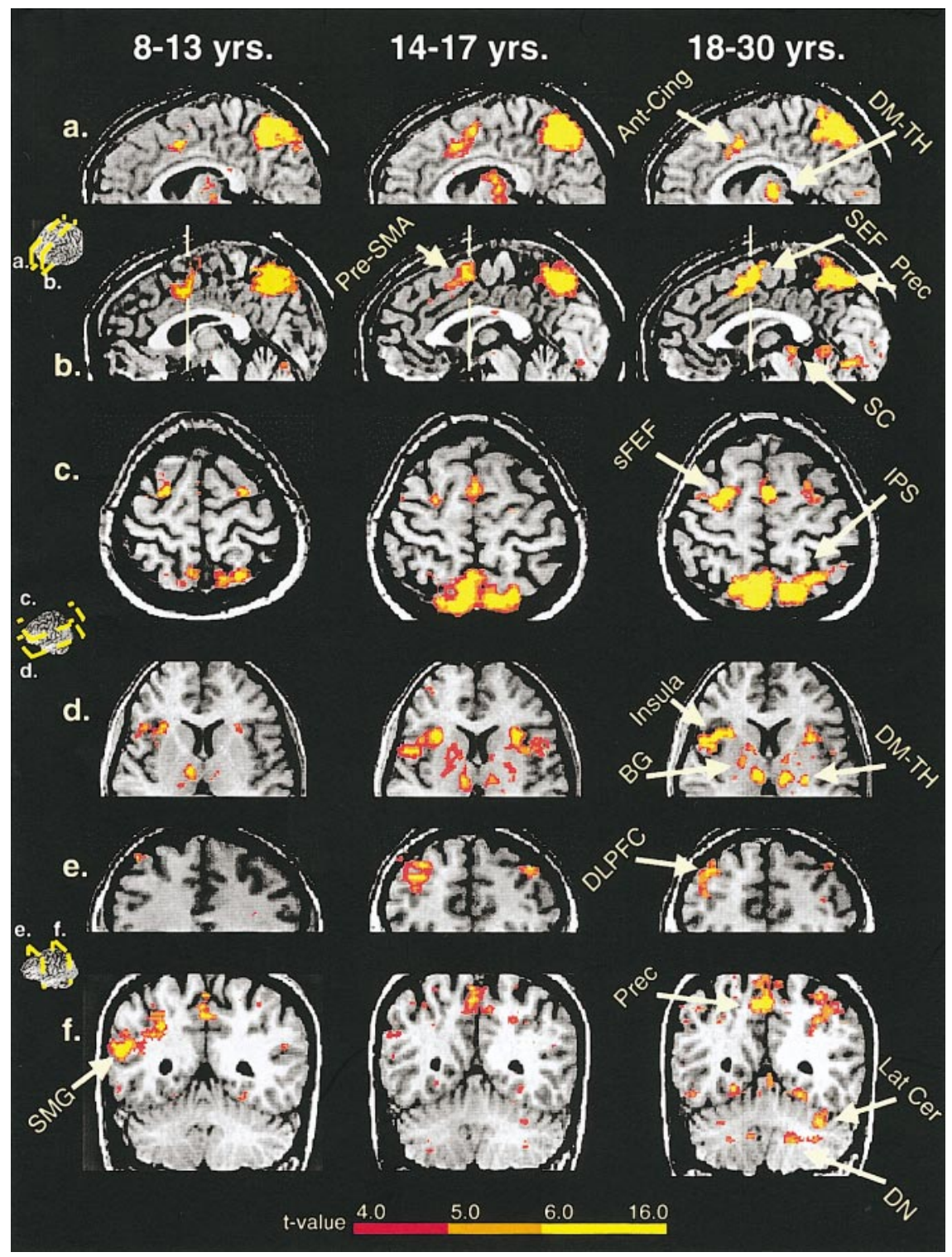

FIG. 2. Group activation maps ( $t \geq 4.0$ ) during an antisaccade task relative to a visually guided prosaccade task superimposed on the structural anatomic image of a representative subject (26 y.o. F) warped into Talairach space. Columns show the average activation for each age group. Rows depict the orientation (rows 1 and $2=$ sagittal; 3 and $4=$ axial; 5 and $6=$ coronal) that optimally illustrate activation in brain regions of interest. Ant-Cing, anterior cingulate; DM-TH, dorsomedial thalamus; Pre-SMA, presupplementary motor area; SEF, supplementary eye fields; Prec, precuneus; SC, superior colliculus; SFEF, superior precentral sulcus aspect of the frontal eye field; IPS, intraparietal sulcus; BG, basal ganglia; DLPFC, dorsolateral prefrontal cortex; SMG, supramarginal sulcus; Lat Cer, Iateral cerebellum; and DN, dentate nucleus.

analyses were performed to compare the percentage signal change in ROI between groups. We also explored the associations between age as a continuous variable and activation in ROI through curve-fitting regression analyses. The threshold value of 4.0 for the t statistic was used because it has yiel ded a reasonable empirical error rate over many studies that our group, as well as other investigators, have performed with our particular scanner and single-shot echoplanar pulse sequence.
We used Analysis of Functional Neurol mages (AF NI) software (Cox, 1996) to overlay the functional data onto co-planar anatomic images. Each individual subject's data were smoothed with a 5.6-mm fullwidth- half-maximum filter and transformed into Talairach space. Then data were averaged across subjects in each age group. AFNI was also used for defining ROIs, as described above, and for 3-D motion correction. AF NI was used to perform voxelwise group comparisons at a $t$ value $\geq 3.0$. This analysis yielded the 


\section{TABLE 1}

Proportion of ROI Activated at a t Value $\geq 4.0$ for Each Group Map

\begin{tabular}{|c|c|c|c|c|c|c|}
\hline \multirow[b]{2}{*}{$\begin{array}{l}\text { ROI } \\
\text { FEF }\end{array}$} & \multicolumn{2}{|c|}{$\begin{array}{c}\text { Children } \\
\text { (8-13 years old) } \\
N=11\end{array}$} & \multicolumn{2}{|c|}{$\begin{array}{c}\text { Adolescents } \\
(14-17 \text { years old }) \\
\mathrm{N}=15\end{array}$} & \multicolumn{2}{|c|}{$\begin{array}{c}\text { Adults } \\
\text { (18-30 years old) } \\
\mathrm{N}=10\end{array}$} \\
\hline & Left & Right & Left & Right & Left & Right \\
\hline SupPCS & $4 \%$ & $4 \%$ & $6 \%$ & $16 \%$ & $13 \%$ & $20 \%$ \\
\hline InfPCS & $0.4 \%$ & $14 \%$ & $13 \%$ & $24 \%$ & $8 \%$ & $20 \%$ \\
\hline Pre-SMA & 0 & $1 \%$ & $9 \%$ & $19 \%$ & $6 \%$ & $7 \%$ \\
\hline SEF & 0 & $13 \%$ & $3 \%$ & $23 \%$ & $15 \%$ & $20 \%$ \\
\hline DLPFC & 0 & $1 \%$ & $4 \%$ & $12 \%$ & $1 \%$ & $7 \%$ \\
\hline IPS & $20 \%$ & $30 \%$ & $40 \%$ & $46 \%$ & $46 \%$ & $29 \%$ \\
\hline Precuneus & $44 \%$ & $65 \%$ & $57 \%$ & $71 \%$ & $39 \%$ & $67 \%$ \\
\hline SMG & $1 \%$ & $42 \%$ & $2 \%$ & $30 \%$ & 0 & $6 \%$ \\
\hline Ant-Cing & $2 \%$ & $8 \%$ & $8 \%$ & $15 \%$ & $6 \%$ & $9 \%$ \\
\hline Insula & $3 \%$ & $12 \%$ & $11 \%$ & $13 \%$ & $6 \%$ & $16 \%$ \\
\hline$B G$ & $.2 \%$ & $2 \%$ & $9 \%$ & $12 \%$ & $6 \%$ & $8 \%$ \\
\hline Thalamus & $11 \%$ & $21 \%$ & $19 \%$ & $28 \%$ & $15 \%$ & $27 \%$ \\
\hline Lat Cer & $3 \%$ & $3 \%$ & $4 \%$ & $1 \%$ & $29 \%$ & $14 \%$ \\
\hline Dentate & 0 & 0 & 0 & 0 & $10 \%$ & $4 \%$ \\
\hline $\begin{array}{l}\text { Nucleus } \\
\text { SC }\end{array}$ & 0 & 0 & 0 & $10 \%$ & $44 \%$ & $10 \%$ \\
\hline
\end{tabular}

Note ROI, region of interest; FEF, frontal eye field; supPCS, superior precentral sulcus; infPCS, inferior precentral sulcus; PreSMA, presupplementary motor area; SEF, supplementary eye fields; DLPFC, dorsolateral prefrontal cortex; IPS, intraparietal sulcus; SMG, supramarginal gyrus; Ant-Cing, anterior cingulate; BG, basal ganglia; Lat Cer, lateral cerebellum; SC, superior colliculus.

number of voxels in each $\mathrm{ROI}$ reflecting significant group differences.

\section{RESULTS}

Behavioral results demonstrated that the prosaccade error rate in the antisaccade task decreased significantly with increasing age (Multiple $\mathrm{R}=0.65$; $\mathrm{P}<$ 0.001 , using an inverse curve fit), consistent with previous findings (Fischer et al., 1997; Munoz et al., 1998). The peak velocity, accuracy, amplitude, duration, and latency of antisaccades did not change significantly with age demonstrating the early maturation of basic sensorimotor processing.

Figure 2 depicts the brain activation during antisaccade task performance for each age group. All groups demonstrated activation in the FEF, SEF, pre-SMA, DLPFC, anterior cingulate, precuneus, IPS, SMG, insula, basal ganglia, and thalamus. Adults showed additional activity in thelateral cerebellar cortex, dentate nucleus of the cerebellum, and superior colliculus. As depicted in Table 1, the proportion of each ROI activated in the group maps indicates that children had the highest activation among all groups in the SMG. Adolescents showed the most activated voxels, in comparison to the other age groups, in the inferior aspect of the FEF, pre-SMA, and DLPFC. The number of activated voxels in the superior aspect of the FEF, lateral cerebellum, and superior colliculus was most robust in adults.

Multivariate (group, task, hemisphere, and ROI) tests on differences in percentage signal change revealed a main effect for group $\left(\mathrm{F}_{2,33}=6.17, \mathrm{P}<0.05\right)$ and ROI $\left(F_{13,21}=21.60, P<0.0001\right)$. Significant interactions were present for task by group $\left(\mathrm{F}_{2,33}=3.953\right.$, $\mathrm{P}<0.05)$, task by ROI $\left(\mathrm{F}_{13,21}=5.43, \mathrm{P}<0.0001\right)$, hemisphere by task by group $\left(F_{2,33}=3.44, P<0.05\right)$, and hemisphere by ROI $\left(F_{13,21}=4.00, P<0.01\right)$.

Regression analyses were performed in order to examine the association of age as a continuous variable with percent signal change at each ROI. Analyses revealed significant associations between age and activation in bilateral intraparietal sulcus and right FEF in both the antisaccade vs prosaccade comparison (left-IPS $=\mathrm{F}_{31}=$ 6.82, $\mathrm{P}<0.05$; right-IPS $=\mathrm{F}_{29}=4.03, \mathrm{P}<0.05$; right$\left.\mathrm{FEF}=\mathrm{F}_{30}=5.93, \mathrm{P}<0.01\right)$ and the prosaccade vs fixation comparison (left-IPS $=\mathrm{F}_{31}=6.03, \mathrm{P}<0.05$; right-IPS $=\mathrm{F}_{29}=4.37, \mathrm{P}<0.05$; right-FEF $=\mathrm{F}_{30}=9.97$, $\mathrm{P}<0.0001$ ), indicating that these areas may be reflecting both the maturation of response suppression and basic sensorimotor and attention systems. Activation in the left lateral cerebellum was found to increase with age only in the antisaccade vs prosaccade comparison $\left(\mathrm{F}_{29}=\right.$ $3.42, \mathrm{P}<0.05$ ), indicating that this area is specific to the maturation of voluntary response suppression. Similar results were found when analyses were performed on the number of significantly activated voxels. The number of activated voxels increased with age in the left lateral cerebellum $\left(\mathrm{F}_{(1,33)}=6.10, \mathrm{P}<0.05\right)$. Additionally, posthoc group comparisons showed that adults had significantly more activated voxels than children in the left dentate nucleus $\left(\mathrm{t}_{19}=2.30, \mathrm{P}<0.05\right)$.

In addition to group comparisons of ROIs, we also performed voxel wise comparisons. Voxel wise group comparisons demonstrated that children had a significantly higher percentage signal change than adults in the right SMG. Adolescents had a significantly higher percentage signal change than either children or adults in right DLPFC. Adults had significantly higher percentage signal changes than both younger groups in the superior FEF (bilaterally in comparison to children, and in the left FEF in comparison to adolescents), and in left thalamus, left IPS, left lateral cerebellum, left dentate nucleus, and the superior colliculus. There were no gender differences in the number of voxel s activated. Activation was greater in the right hemisphere in the antisaccade task in regions induding: superior precentral sulcus, SE F, insula, precuneus, anterior cingulate, and SMG.

\section{DISCUSSION}

\section{Brain Regions Subserving Antisaccades}

Consistent with prior neuroimaging studies, our results indicate that the antisaccade task activates the 
FEF, SEF, DLPFC, posterior parietal cortex, anterior cingulate, striatum, and thalamus, more robustly than do prosaccades (Schlag-Rey et al., 1997; Guitton et al., 1985; Sweeney et al., 1996). Activation in the posterior parietal cortex was found within the IPS, in the precuneus, and in the SMG of the inferior parietal lobe. We also found activation in the insula, pre-SMA, lateral cerebellar cortex, dentate nucleus, and superior colliculus, which, to our knowledge, has not been reported previously in humans in an antisaccade task.

Activation in the DLPFC, anterior cingulate, and intraparietal sulcus reflects the executive functions attributed to these regions, including maintaining a cognitive plan to initiate stop signals (Rowe et al., 2000; Funahashi et al., 1993). Activation in SC and FEF probably reflect the metabolic demands of inhibitory processes that according to Munoz et al. (1998), are necessary to dampen the activity of saccade-generating pyramidal neurons when subjects anticipate the need to suppress an impending saccade.

\section{Developmental Changes}

Our results demonstrate both great overlap and qualitative differences in the pattern and extent of brain activation across age groups paralleling the enhanced voluntary control of behavior throughout adolescence. The presence of robust activation in several cortical regions in children indicates that these regions already participate in voluntary response suppression in childhood. Similar results have been shown in developmental fMRI studies of response suppression (Casey et al., 1997) and nonspatial working memory tasks (Thomas et al., 1999). Our study indicated that activation in SEF, insula, precuneus, and anterior cingulate did not show developmental changes during the antisaccade task, suggesting that children, like older subjects, were accessing these elements of basic oculomotor and attention circuitry that enable voluntary saccadic responses.

Both children and adolescents demonstrated less activation than adults in the superior FEF, IPS, thalamus, lateral cerebellum, dentate nucleus of the cerebellum, and the superior colliculus. These regions may form part of a widely distributed network subserving antisaccade responses. Preparatory attenuation of saccade-related neuronal activity in the SC (Everling et al., 1999) and FEF (Everling et al., 2000) has been found to underlie the ability to successfully suppress a saccade during antisaccade tasks. The cerebellum, via thalamic inputs to prefrontal and premotor areas (Middleton et al., 1994; Kim et al., 1994), may be involved in establishing the task-appropriate inhibitory preparatory activity necessary to successfully suppress automatic behavioral responses (Deiber et al., 1996). The relatively late integration of these widely distributed regions into this brain network may be an impor- tant basis for the ability of adults to optimize the preparedness required to consistently suppress prepotent responses on antisaccade tasks.

As evident in the group maps, in adolescence and adulthood, in comparison to younger children, we observed activation in the basal ganglia. The appearance of significant activation in the striatum in adolescence may represent a first step in the maturation of corticostriato-thalamocortical loops (Alexander et al., 1986) that appear to be important for maintaining stable anticipatory response sets and generating voluntary behavior. Children, in contrast, showed increased activation in the SMG, perhaps reflecting a reliance on visuospatial processing to compensate for immature access to widely distributed regions required to maintain appropriate response sets. Adolescents demonstrated greater activation than both children and adults in the DLPFC, indicating their greater reliance on executive prefrontal behavior control systems. This activation in the DLPFC, possibly facilitated by increased functional integrity of cortico-striato-thalamocortical loops, could provide inhibitory inputs to FEF and SEF to improve performance on the antisaccade task until the maturation of widely distributed function-especially the integration of cerebellar inputscan sustain adult-level suppression of prepotent responses. Unlike the Casey et al. (1997) results indicating increased activation of prefrontal cortex in children compared to adults during a Go-No-Go task, we found increased prefrontal activation in adolescents. This is not surprising since performance in the Go-No-Go task matures earlier (at approximately 12 years of age - Levin et al., 1991) than in the antisaccade task (15-20 years of age-Fischer et al., 1997; Munoz et al., 1998). The difficulty of the antisaccade task may preclude the efficient use of DLPFC in our youngest subjects who often made many errors performing the task. Adolescents may have recruited DLPFC at high levels, as did the children in the Casey et al. (1997) study, because of the greater difficulty performing the antisaccade task correctly.

Data from our whole brain fMRI study provide provocative new evidence indicating that the development of the ability to voluntarily initiate and suppress be havior in humans is influenced by the maturation of integrated function among the neocortex, striatum, thalamus, and cerebellum. Synaptic pruning and myelination during childhood and adolescence are important for enhancing widely distributed brain functions by refining synaptic connections and enhancing the transfer of information throughout the brain in a rapid manner. Such maturational changes may help establish and maintain patterns of neuronal activity needed for working memory and response preparedness. On the other hand, the immature functional connectivity of the child's brain could make it difficult to integrate the function of many distant brain regions, thus ham- 
pering the ability to maintain states of preparedness that facilitate the voluntary suppression of reflexive behavior and the generation of adaptive context-appropriate voluntary responses.

\section{ACKNOWLEDGMENTS}

This study was supported by MH01727, MH01433, NARSAD, HD35469, NS35949, and MH45156. We thank the staff at the Neurobehavioral Studies program and UPMC MR Center for assistance in testing subjects. We thank Dawn Boarts for assistance in recruitment and testing of subjects. We also thank Drs. David Kupfer and David Lewis for valuable comments on the findings reported in this manuscript.

\section{REFERENCES}

Alexander, G. E., Delong, M. R., and Strick, P. L. 1986. Parallel organization of functionally segregated circuits linking basal ganglia and cortex. Annu. Rev. Neurosci. 9: 357-381.

Bjorklund, D. F., and Harnishfeger, K. K. 1990. The resources construct in cognitive devel opment: Diverse sources of evidence and a theory of inefficient inhibition. Dev. Rev. 10: 48-71.

Bourgeois, J . P. 1993. Synaptogenesis in the Prefrontal Cortex of the Macaque. In Developmental Neurocognition: Speech and Face Processing in the First Year of Life (B. de Boysson-Bardies, Ed.), pp. 31-39. Kluwer, The Netherlands.

Burman, D. D., and Bruce, C. J . 1997. Suppression of task-related saccades by electrical stimulation in the primate's frontal eye field. J . Neurophysiol. 77: 2252-2267.

Casey, B. J ., Trainor, R. J ., Orendi, J . L., Schubert, A. B., Nystrom, L. E., Giedd, J. N., Astellanos, F. X., Haxby, J. V., Noll, D. C., Cohen, J . D., Forman, S. D., Dahl, R. E., and Rapoport, J . L. 1997. A developmental functional MRI study of prefrontal activation during performance of a go-no-go task. J . Cogn. Neurosci. 9: 835847.

Caviness, V. S., Kennedy, D. N., Bates, J . F., and Makris, N. 1996. The Developing Human Brain: A Morphometric Profile. In Developmental Neuroimaging: Mapping The Development of Brain and Behavior (R. W. Thatcher, G. Reid Lyon, J. Rumsey, and N. A. Krasnegor, Eds.), pp. 3-14. Academic Press, New York.

Changeux, J.-P., and Danchin, A. 1976. Selective stabilisation of developing synapses as a mechanism for the specification of neuronal networks. Nature 264: 705-712.

Chugani, H. T. 1998. A critical period of brain development: Studies of cerebral glucose utilization with PET. Prev. Med. 27: 184-188.

Cohen, M. S. 1997. Parametric analysis of fMRI data using linear systems methods. Neurol mage 6: 93-103.

Corbetta, M., Kincade, J. M., Ollinger, J. M., McAvoy, M. P., and Shulman, G. L. 2000. Voluntary orienting is dissociated from target detection in human posterior parietal cortex. Nature Neurosci. 3, 292-297.

Cox, R. W. 1996. AFNI: Software for analysis and visualization of functional magnetic resonance neuroimages. Comput. Biomed. Res. 29: 162-173.

Deiber, M. P., I banez, V., Sadato, N., and Hallett, M. 1996. Cerebral structures participating in motor preparation in humans: A positron emission tomography study. J . Neurophysiol. 75: 233247.

Dempster, F. N. 1993. Resistance to Interference: Developmental changes in a basic processing mechanism. In Emerging Themes in Cognitive Devel opment, Volumel: Foundations (M. L. Howe and R. Pasnak, Eds.), pp. 3-27. Springer-Verlag, New York.
Doricchi, F., Perani, D., I ncoccia, C., Grassi, F., Cappa, S. F., Bettinardi, V., Galati, G., Pizzamiglio, L., and Fazio, F. 1997. Neural control of fast-regular saccades and antisaccades: An investigation using positron emission tomography. Exp. Brain Res. 116: 50-62.

Eddy, W. F., Fitzgerald, M., Genovese, C. R., Mockus, A., and Noll, D. C. 1996. Functional image analysis software-Computational olio. In Proceedings in Computational Statistics (A. Prat, Ed.), pp. 39-49. Physica-Verlag, Heidel berg.

Everling, S., Dorris, M. C., Klein, R. M., and Munoz, D. P. 1999. Role of primate superior colliculus in preparation and execution of anti-saccades and pro-saccades. J . Neurosci. 19: 2740-2754.

Everling, S., and Munoz, D. P. 2000. Neuronal correlates for preparatory set associated with pro-saccades and anti-saccades in the primate frontal eye field. J . Neurosci. 20: 387- 400.

Fischer, B., Biscaldi, M., and Gezeck, S. 1997. On the development of voluntary and reflexive components in human saccade generation. Brain Res. 754: 285-297.

Funahashi, S., Chafee, M. V., and Goldman-Rakic, P. S. 1993. Prefrontal neuronal activity in rhesus monkeys performing a delayed anti-saccade task. Nature 365: 753-758.

Giedd, J. N., Blumenthal, J ., J effries, N. O., Castellanos, F. X., Liu, H., Zijdenbos, A., Paus, T., Evans, A. C., and Rapoport, J . L. 1999. Brain development during childhood and adolescence: A longitudinal MRI study. Nature Neurosci. 2: 861-863.

Goldman-Rakic, P. S. 1988. Topography of cognition: Parallel distributed networks in primate association cortex. Annu. Rev. Neurosci. 11: 137-156.

Gottlieb, J ., and Goldberg, M. E. 1999. Activity of neurons in the lateral intraparietal area of the monkey during an antisaccade task. Nature Neurosci. 2: 906-912.

Guitton, D., Buchtel, H. A., and Douglas, R. M. 1985. Frontal lobe lesions in man cause difficulties in suppressing reflexive glances and in generating goal-directed saccades. Exp. Brain Res. 58: 455- 472.

Hallett, P. E. 1978. Primary and secondary saccades to goals defined by instructions. Vision Res. 18: 1279-1296.

Huttenlocher, P. R. 1990. Morphometric study of human cerebral cortex development. Neuropsychologia 28: 517-527.

Kastner, S., Weerd, P. D., Desimone, R., and Ungerleider, L. G. 1988. Mechanisms of directed attention in the human extrastriate cortex as revealed by functional MRI. Science 282: 108-111.

Kim, S. G., Ugurbil, K., and Strick, P. L. 1994. Activation of a cerebellar output nucleus during cognitive processing. Science 265: 949-951.

Levin, H. S., Culhane, K. A., Hartmann, J ., Evankovich, K., and Mattson, A. J . 1991. Developmental changes in performance on tests of purported frontal lobe functioning. Dev. Neuropsych. 7: 377-395.

Mesulam, M. M. 1981. A cortical network for directed attention and unilateral neglect. Ann. Neurol. 10: 309-325.

Middleton, F. A., and Strick, P. L. 1994. Anatomical evidence for cerebellar and basal ganglia involvement in higher cognitive function. Science 266: 458-461.

Minshew, N. J ., Luna, B., and Sweeney, J. A. 1999. Oculomotor evidence for neocortical systems but not cerebellar dysfunction in autism. Neurology 52: 917-922.

Munoz, D. P., Broughton, J ., Goldring, J ., and Armstrong, I. 1998. Age-related performance of human subjects on saccadic eye movement tasks. Exp. Brain Res. 217: 1-10.

Muri, R. M., Heid, O., Nirkko, A. C., Ozdoba, C., Felblinger, J., Schroth, G., and Hess, C. W. 1998. Functional organization of saccades and antisaccades in the frontal lobe in humans: A study with echo planar functional magnetic resonance imaging. J . Neurol. Neurosurg. Psychiatry 65: 374-377. 
O’Driscoll, G. A., Alpert, N. M., Matthysse, S. W., Levy, D. L., Rauch, S. L., and Holzman, P. S. 1995. Functional neuroanatomy of antisaccade eye movements investigated with positron emission tomography. Proc. Natl. Acad. Sci. USA 92: 925-929.

Paus, T., Zijdenbos, A., Worsley, K., Collins, D. L., Blumenthal, J ., and Evans, A. C. 1999. Structural maturation of neural pathways in children and adolescents: In vivo study. Science 283: 19081911.

Petit, L., Clark, V. P., Ingeholm, J ., and Haxby, J . V. 1997. Dissociation of saccade-related and pursuit-related activation in human frontal eye fields as revealed by fMRI . J . Neurophysiol. 77: 33863390.

Petit, L., and Haxby, J. 1999. Functional anatomy of pursuit eye movements in humans as revealed by fMRI. J . Neurophysiol. 82: 463- 471.

Picard, N., and Strick, P. L. 1997. Activation on the medial wall during remembered sequences of reaching movements in monkeys. J . Neurophysiol. 77: 2197-2201.

Rakic, P. 1995. The Development of the Frontal Lobe: A View from the Rear of the Brain. In Epilepsy and the Functional Anatomy of the Frontal Lobe (H. H. J asper, S. Riggio, and P. S. GoldmanRakic, Eds.), pp. 1- 8. Raven Press, New York.

Rosenberg, D. R., Sweeney, J . A., Gillen, J ., Kim, J ., Varenelli, M., O'Hearn, K., Erb, P., Davis, D., and Thulborn, K. 1997. Magnetic resonance imaging of children without sedation: Preparation with simulation. J. Am. Acad. Child Adolesc. Psychiatry 36: 853- 859.

Rowe, J . B., Toni, I., J osephs, O., Frackowiak, R. S. J ., and Passingham, R. E. 2000. The prefrontal cortex: Response selection or maintenance within working memory? Science 288: 1656-1660.

Schlag-Rey, M., Amador, N., Sanchez, H., and Schlag, J. 1997. Antisaccade performance predicted by neuronal activity in the supplementary eye field. Nature 390: 398-400.

Sowell, E. R., Thompson, P. M., Holmes, C. J ., J ernigan, T. L., and Toga, A. W. 1999. In vivo evidence for post-adolescent brain maturation in frontal and striatal regions. Nature Neurosci. 2: 859861.

Sweeney, J . A., Mintun, M. A., Kwee, S., Wiseman, M. B., Brown, D. L., Rosenberg, D. R., and Carl, J . R. 1996. A positron emission tomography study of voluntary saccadic eye movements and spatial working memory. J. Neurophysiol. 75: 454- 468.

Thatcher, R. W., Walker, R. A., and Giudice, S. 1987. Human cerebral hemispheres develop at different rates and ages. Science $\mathbf{2 3 6}$ 1110-1113.

Thomas, K. M., King, S. W., Franzen, P. L., Welsh, T. F., Berkowitz, A. L., Noll, D. C., Birmaher, V., and Casey, B. J . 1999. A developmental functional MRI study of spatial working memory. NeuroImage 10: 327-338.

Yakovlev, P. I., and Lecours, A. R. 1967. Regional Development of the Brain in Early Life Blackwell Scientific, Oxford. 